IJMMS 2003:5, 295-304

PII. S0161171203111325

http://ijmms.hindawi.com

(c) Hindawi Publishing Corp.

\title{
CHARACTERIZATIONS OF VECTOR-VALUED WEAKLY ALMOST PERIODIC FUNCTIONS
}

\author{
CHUANYI ZHANG
}

Received 10 November 2001

\begin{abstract}
We characterize the weak almost periodicity of a vector-valued, bounded, continuous function. We show that if the range of the function is relatively weakly compact, then the relative weak compactness of its right orbit is equivalent to that of its left orbit. At the same time, we give the function some other equivalent properties.
\end{abstract}

2000 Mathematics Subject Classification: 43A60, 43A07.

1. Introduction. Let $S$ be a semitopological semigroup, let $\mathscr{X}$ be a Banach space, and let $\mathscr{C}(S, \mathscr{X})$ be the space of bounded continuous functions from $S$ to $\mathscr{X}$ with supremum norm. Let $f \in \mathscr{C}(S, \mathscr{X})$. The right (left) translate of $f$ by $s \in S$ is the function $R_{S} f\left(L_{S} f\right)$ such that $R_{S} f(t)=f(t s)$ and $\left(L_{S} f(t)=f(s t)\right)$ for all $t \in S$. The function $f$ is said to be weakly almost periodic if its right orbit $R_{S} f=\left\{R_{S} f: s \in S\right\}$ is relatively weakly compact in $\mathscr{C}(S, \mathscr{X})$. We denote by $\mathscr{W} \mathscr{A} \mathscr{P}(S, \mathscr{X})$ all such functions.

In the case that $\mathscr{X}=\mathbb{C}$, the complex number field, we will omit $\mathscr{X}$ from our notations and write, for example, $\mathscr{C}(S)$ for $\mathscr{b}(S, \mathbb{C})$.

Recently, some authors have investigated $\mathscr{W} \mathscr{A} \mathscr{P}(S, \mathscr{X})$ and exploited its applications in many areas $[1,2,4,5,6,7,8,9]$. However, some questions remain unsolved. For example, [2, Theorems 4.2 .3 and 4.2.6] give a number of equivalent properties for a function $f \in \mathscr{C}(S)$ to be weakly almost periodic. It is natural to ask if the similar equivalent properties are true for a function in $\mathscr{C}(S, \mathscr{C})$. In this paper, we investigate these problems and give positive answers.

It is shown in [4, Proposition 2.8] that the equivalence of relative weak compactness for $R_{S} f$ and the left orbit $L_{S} f=\left\{L_{S} f: s \in S\right\}$ holds if $S$ admits an identity and the range $f(S)$ is relative compact in $\mathscr{X}$. We will give an example at the end of the paper to show that the assumptions both on $S$ and on $f(S)$ are not essential to get the equivalence. We will show the equivalence under the assumption that $f(S)$ is relatively weakly compact. At the same time, we characterize a vector-valued weakly almost periodic function by giving it as many equivalent properties as a scalar-valued weakly almost periodic function has. We will not assume that a semitopological semigroup $S$ admits an identity. In fact, if $S$ has an identity, we can drop the condition $f(S)$, being either relative norm compact or relatively weakly compact (see Remark 3.6(b)). 
2. Vector-valued means. To show the main results of the paper in Section 3, we need some facts of vector-valued means. Unless otherwise mentioned, all the results of this section come from [8, Sections 2 and 3].

Let $\mathscr{L}, 9$ be two normed linear spaces and let $y^{*}$ be the dual space of $\mathscr{Y}$. Let $\mathscr{L}(\mathscr{L}, \mathscr{Y})$ be the space of bounded linear operators from $\mathscr{E}$ to $\mathscr{Y}$. With the norm topology, $\mathscr{L}(\mathscr{L}, \mathscr{y})$ is a Banach space. We can also furnish $\mathscr{L}(\mathscr{L}, \mathscr{Y})$ with the following two topologies, both of them make $\mathscr{L}(\mathscr{L}, \mathscr{Y})$ a locally convex topological space [3, VI.1.2, VI.1.3]:

(1) the strong operator topology $\tau_{s}$, which is the weakest topology of $\mathscr{L}(\mathscr{L}, y)$ relative to which the mapping $\mu \rightarrow \mu(z): \mathscr{L}(\mathscr{L}, \mathscr{y}) \rightarrow \mathscr{Y}$, is continuous for each $z \in \mathscr{L}$;

(2) the weak operator topology $\tau_{w}$, which is the weakest topology of $\mathscr{L}(\mathscr{L}, \mathscr{y})$ relative to which the mapping $\mu \rightarrow y^{*}[\mu(z)]: \mathscr{L}(\mathscr{L}, \mathscr{y}) \rightarrow \mathbb{C}$, is continuous for each $z \in \mathscr{L}$ and $y^{*} \in \mathscr{Y}^{*}$.

For $\mathscr{L}\left(\mathscr{L}, y^{*}\right)$, we have the following topology that also makes $\mathscr{L}\left(\mathscr{L}, y^{*}\right)$ a locally convex topological space [3, page 476]:

(3) the weak* operator topology $\tau_{w^{*}}$, which is the weakest topology of $\mathscr{L}\left(\mathscr{L}, \mathscr{Y}^{*}\right)$ relative to which the mapping $\mu \rightarrow[\mu(z)](y): \mathscr{L}\left(\mathscr{L}, y^{*}\right) \rightarrow \mathbb{C}$, is continuous for each $z \in \mathscr{E}$ and $y \in \mathscr{Y}$.

Let $S$ be a nonempty set, let $\mathscr{X}$ be a Banach space, and let $\mathscr{B}(S, \mathscr{X})$ be the space of bounded functions from $S$ to $\mathscr{X}$ with supremum norm. Let $\mathscr{A}$ be a subspace of $\mathscr{B}(S, \mathscr{X})$ containing the constant functions.

DEFinition 2.1. A mean $\mu$ on $\mathscr{A}$ is a linear operator from $\mathscr{A}$ to $\mathscr{X}$ such that $\mu(f) \in \overline{\operatorname{co}} f(S)$ for all $f \in \mathscr{A}$, denoted by $M(\mathscr{A})$ of all means on $\mathscr{A}$.

We define the evaluation mapping $\epsilon: S \rightarrow M(\mathscr{A})$ as follows: for $s \in S, \epsilon(s) f=$ $f(s), f \in \mathscr{A}$. The following proposition comes from [9, Propositions 1.5 and 1.6].

Proposition 2.2. Let $\mathscr{A}$ be a subspace of $\mathscr{B}(S, \mathscr{X})$ containing the constant functions. Then for both $\tau_{s}$ and $\tau_{w}, M(\mathscr{A})$ is convex and closed in $\mathscr{L}(\mathscr{A}, \mathscr{X})$, and $\operatorname{co}(\epsilon(S))$ is dense in $M(\mathscr{A})$. Furthermore, if $\mathscr{A}$ is such that $f(S)$ is relatively weakly compact in $\mathscr{L}$ for all $f \in \mathscr{A}$, then $M(\mathscr{A})$ is $\tau_{w}$-compact.

We embed $\mathscr{X}$ into its double dual space $\mathscr{L}^{* *}$ canonically and let $\iota(\mathscr{L})$ denote its canonical image in $\mathscr{X}^{* *}$; similarly, we embed $f(S)$ into $\mathscr{X}^{* *}$ for every $f \in \mathscr{A}$ and get a subspace $\iota(\mathscr{A})$ of $\mathscr{B}(S, \iota(\mathscr{X}))$. A function of $\mathscr{A}$ may be regarded as a function of $\iota(\mathscr{A})$, and vice versa. Replacing $\mathscr{A}$ and $\mathscr{X}$ by $\iota(\mathscr{A})$ and $\mathscr{X}^{* *}$ in Definition 2.1, respectively, we get $M(\iota(\mathscr{A}))$. A mean of $M(\mathscr{A})$ may be regarded as a mean of $M(\iota(\mathscr{A}))$, and vice versa. This leads to the following more general definition of means.

DEFINITION 2.3. Let $\mathscr{A}$ be a subspace of $\mathscr{B}\left(S, \mathscr{L}^{* *}\right)$. A linear map $\mu: \mathscr{A} \rightarrow \mathscr{L}^{* *}$ is called a $w^{*}$ mean on $\mathscr{A}$ provided $\mu(f) \in \overline{\mathrm{co}} w^{*} f(S)$, for all $f \in \mathscr{A}$, where $w^{*}$ stands for the weak* topology $\sigma\left(\mathscr{L}^{* *}, \mathscr{L}^{*}\right)$. Denote by $w^{*} M(\mathscr{A})$ the set of 
all $w^{*}$ means on $\mathscr{A}$. In the case that $\mathscr{A} \subset \mathscr{B}(S, \mathscr{X})$, we define $w^{*} M(\mathscr{A})$ to be $w^{*} M(\iota(\mathscr{A}))$.

Both Definitions 2.1 and 2.3 will reduce to the definition of a scalar-valued mean when $\mathscr{X}=\mathbb{C}[1,2.1 .2]$.

Proposition 2.4. Let $\mathscr{A}$ be a linear subspace of $\mathscr{B}\left(S, \mathscr{L}^{* *}\right)$. Then for $\tau_{w^{*}}$

(1) $w^{*} M(A)$ is convex and compact;

(2) let $\epsilon$ be the evaluation map $S \rightarrow w^{*} M(\mathscr{A})$, then $\operatorname{co}(\epsilon(S))$ is dense in $w^{*} M(\mathscr{A})$.

Proposition 2.5. Every member of $w^{*} M(\mathscr{A})$ can be extended to a member of $w^{*} M\left(\mathscr{B}\left(S, \mathscr{X}^{* *}\right)\right)$.

We call the scalar-valued function space

$$
\mathscr{F}=\operatorname{sp}\left\{[f(\cdot)]\left(x^{*}\right): x^{*} \in \mathscr{L}^{*}, f \in \mathscr{A}\right\}
$$

generated space of $\mathscr{A}$.

Proposition 2.6. Let $\mathscr{A}$ be a linear subspace of $\mathscr{B}\left(S, \mathscr{L}^{* *}\right)$ containing the constant functions, and let $\mathscr{F}_{\mathrm{F}}$ be its generated space. Then, there is an isometric $\tau_{w^{*}-\sigma}\left(\mathscr{F}^{*}, \mathscr{F}\right)$ homeomorphism $\mu \rightarrow \varphi_{\mu}: w^{*} M(\mathscr{A}) \rightarrow M(\mathscr{F})$ such that

$$
[\mu(f)]\left(x^{*}\right)=\varphi_{\mu}\left[f(\cdot)\left(x^{*}\right)\right] \quad\left(f \in \mathscr{A}, x^{*} \in \mathscr{L}^{*}\right) .
$$

3. Main results. A nonempty set $S$ that is a semigroup and also a topological space is called a semitopological semigroup provided that the maps $s \rightarrow t s$ and $s \rightarrow s t$ from $S$ to $S$ are continuous for all $t \in S$. Let $S$ be such a set, and let $\mathscr{A}$ be a subspace of $\mathscr{C}(S, \mathscr{X})$. We say $\mathscr{A}$ is right (resp., left) translation invariant if $R_{S} \mathscr{A}=\left\{R_{S} f: s \in S, f \in \mathscr{A}\right\} \subset \mathscr{A}$ (resp., $L_{S} \mathscr{A}=\left\{L_{S} f: s \in S, f \in \mathscr{A}\right\} \subset \mathscr{A}$ ). We say $\mathscr{A}$ is translation invariant if it is both right and left translation invariant.

Let $\mathscr{A}$ be a translation invariant subspace of $\mathscr{C}(S, \mathscr{X})$. For $\mu \in M(\mathscr{A})$, define $T_{\mu}: \mathscr{A} \rightarrow \mathscr{B}(S, \mathscr{X})$ by

$$
T_{\mu} f(s)=\mu\left(L_{s} f\right) \quad(f \in \mathscr{A}, s \in S)
$$

and $U_{\mu}: \mathscr{A} \rightarrow \mathscr{B}(S, \mathscr{X})$ by

$$
U_{\mu} f(s)=\mu\left(R_{s} f\right) \quad(f \in \mathscr{A}, s \in S) .
$$

We call $T_{\mu}\left(U_{\mu}\right)$ left (right) introversion operator determined by $\mu$. We will say that $\mathscr{A}$ is left (right) introverted if $T_{\mu} \mathscr{A} \subset \mathscr{A}\left(U_{\mu} \mathscr{A} \subset \mathscr{A}\right)$ for all $\mu \in M(\mathscr{A})$. We will say that $\mathscr{A}$ is introverted if it is both left and right introverted.

Similarly, we define an introversion operator from $\mathscr{A}$ to $\mathscr{B}\left(S, \mathscr{X}^{* *}\right)$ if $\mathscr{A}$ is a translation invariant subspace of $\mathscr{B}\left(S, \mathscr{X}^{* *}\right)$ and $\mu \in w^{*} M(\mathscr{A})$.

To show Theorem 3.2, we need the following proposition that characterizes weak almost periodicity of a function in $\mathscr{C}(S)$. 
Proposition $3.1[2,4.2 .6]$. Let $S$ be a semitopological semigroup, let $f \in$ $\mathscr{C}(S)$, and let $\mathscr{F}=\mathscr{W} \mathscr{A P}(S)$. Then, the following statements are equivalent:

(1) $f \in W \mathscr{A} \mathscr{P}(S)$;

(2) $L_{S} f$ is relatively weakly compact in $\mathscr{C}(S)$;

(3) the mapping $\varphi \rightarrow T_{\varphi} f: M(\mathscr{F}) \rightarrow \mathscr{B}_{B}(S)$ is $\sigma\left(\mathscr{F}^{*}, \mathscr{F}\right) \rightarrow \sigma\left(\mathscr{B}(S), \mathscr{B}(S)^{*}\right)$ continuous;

(4) the mapping $\varphi \rightarrow U_{\varphi} f: M(\mathscr{F}) \rightarrow \mathscr{B}(S)$ is $\sigma\left(\mathscr{F}^{*}, \mathscr{F}\right) \rightarrow \sigma\left(\mathscr{B}(S), \mathscr{B}(S)^{*}\right)$ continuous;

(5) for all $\varphi, \psi \in M(\mathscr{F}), \varphi\left(T_{\psi} f\right)=\psi\left(U_{\varphi} f\right)$.

We will generalize Proposition 3.1 from scalar-valued function to vectorvalued function in the next theorem. We will use some results of the previous section to show the theorem. To make notations short, we let

$$
\iota(\mathscr{C})=\mathscr{C}(S, \iota(\mathscr{X})), \quad \iota(\mathscr{B})=\mathscr{B}(S, \iota(\mathscr{X})), \quad \mathscr{B}=\mathscr{B}\left(S, \mathscr{X}^{* *}\right) .
$$

As in the paragraph before Definition 2.3, $\iota(\mathscr{X})$ is the canonical image of $\mathscr{X}$ in $\mathscr{L}^{* *}$; an $f \in \mathscr{B}(S, \mathscr{L})$ and its corresponding function in $\iota(\mathscr{B})$ will be regarded as same function.

Note that both $\mathscr{B}$ and $\iota(\mathscr{B})$ have the same generated space $\mathscr{B}(S)$, the space of bounded scalar-valued functions on $S$.

THEOREM 3.2. Let $S$ be a semitopological semigroup and let $\mathscr{X}$ be a Banach space. Let $\mathscr{A}=\mathscr{W} \mathscr{A} \mathscr{P}(S, \mathscr{X})$ and let $f \in \mathscr{C}(S, \mathscr{X})$ be such that $f(S)$ is relatively weakly compact in $\mathscr{X}$. Then the following statements are equivalent:

(1) $f \in \mathscr{A}$, that is, $R_{S} f$ is relatively weakly compact in $\mathscr{C}(S, \mathscr{X})$;

(2) $L_{S} f$ is relatively weakly compact in $\mathscr{C}(S, \mathscr{X})$;

(3) the mapping $\mu \rightarrow T_{\mu} f: w^{*} M(\mathscr{B}) \rightarrow \iota(\mathscr{B})$ is $\tau_{w^{*}-\sigma}\left(\iota(\mathscr{B}), \iota(\mathscr{B})^{*}\right)$ continuous;

(4) the mapping $\mu \rightarrow U_{\mu} f: w^{*} M(\mathscr{B}) \rightarrow \iota(\mathscr{B})$ is $\tau_{w^{*}-\sigma} \sigma\left(\iota(\mathscr{B}), \iota(\mathscr{B})^{*}\right)$ continuous;

(5) for all $\mu, \nu \in w^{*} M(\mathscr{B})$,

$$
\mu\left(T_{v} f\right)=v\left(U_{\mu} f\right)
$$

Proof. Since $f(S)$ is relatively weakly compact in $\mathscr{X}$, the functions $T_{\mu} f$ and $U_{\mu} f$ are in $\iota(\mathscr{B})$ for all $\mu \in w^{*} M(\mathscr{B})$.

Let $\epsilon$ and $\epsilon^{\prime}$ be the evaluation mappings on $\mathscr{B}$ and $\mathscr{B}(S)$, respectively. Let $B=\overline{\mathrm{CCO}}^{\top} w^{*} \epsilon(S)$, and let $B_{\mathscr{B}(S)^{*}}$ be the unit ball of $\mathscr{B}(S)^{*}$. By $[2,2.1 .14], B_{\mathscr{B}}(S)^{*}=$ $\overline{\mathrm{CCO}}^{w^{*}} \epsilon^{\prime}(S)$, where $w^{*}$ stands for $\sigma\left(\mathscr{B}(S)^{*}, \mathscr{B}(S)\right)$. It follows from Propositions 2.4(2) and 2.6 that $B$ and $B_{\mathscr{B}}(S)^{*}$ are $\tau_{w^{*}-\sigma} \sigma\left(\mathscr{B}(S)^{*}, \mathscr{B}(S)\right)$ homeomorphic.

Define $V: B \rightarrow \mathscr{B}$ by

$$
V(\mu)=T_{\mu} f
$$


$V$ is continuous from $\tau_{w} *$ to the weak* pointwise convergence topology $P$. For, if $\left\{\mu_{\alpha}\right\} \subset B$ and $\mu \in B$ are such that $\mu_{\alpha} \rightarrow \mu$ in $\tau_{w^{*}}$, then

$$
\begin{aligned}
{\left[T_{\mu_{\alpha}} f(s)\right]\left(x^{*}\right) } & =\left[\mu_{\alpha}\left(L_{S} f\right)\right]\left(x^{*}\right) \longrightarrow\left[\mu\left(L_{S} f\right)\right]\left(x^{*}\right) \\
& =\left[T_{\mu} f(s)\right]\left(x^{*}\right) \quad\left(x^{*} \in \mathscr{X}^{*}, s \in S\right) .
\end{aligned}
$$

Since $B$ is $\tau_{w} *$-compact (Proposition 2.4(1)), we have

$$
V(B)=\overline{\operatorname{cco}}\left(R_{S} f\right),
$$

where closure in $\iota(\mathscr{B})$ is taken in the weak* pointwise convergence topology $P$. Now, we show that (1) implies (3).

By the Krein-Smulian theorem [2, Theorem A.10], $\operatorname{cco}\left(R_{S} f\right)$ is relatively weakly compact in $\iota(\mathscr{C})$, which in view of (3.7) implies that $V(B)$ is $\sigma(\iota(\mathscr{C})$, $\left.\iota(\mathscr{C})^{*}\right)$-closure of $\operatorname{cco}\left(R_{S} f\right)$ in $\iota(\mathscr{C})$ and that $V(B)$ is $\sigma\left(\iota(\mathscr{C}), \iota(\mathscr{C})^{*}\right)$-compact. Therefore, the weak topology $\sigma\left(\iota(\mathscr{C}), \iota(\mathscr{C})^{*}\right)$ and the topology $P$ coincide on $V(B)$. So, $V$ is $\tau_{w} *-\sigma\left(\iota(\mathscr{B}), \iota(\mathscr{B})^{*}\right)$ continuous on $B$.

To show (3), we define, for $\Phi \in \iota(\mathscr{B})^{*}$, the linear functional $\Phi \circ T: \mathscr{B}(S)^{*} \rightarrow \mathbb{C}$ by

$$
\Phi \circ T\left(\varphi_{\mu}\right)=\Phi\left(T_{\mu} f\right)
$$

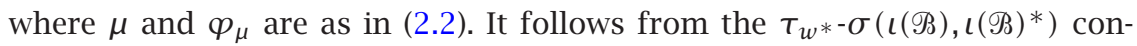
tinuity on $B$ of $V$ that $\Phi \circ T$ is $\sigma\left(\mathscr{B}_{(}(S)^{*}, \mathscr{B}(S)\right)$ continuous on $B_{\mathscr{B}}(S)^{*}$. By Grothendieck's completeness theorem [2, Proposition A.8], $\Phi \circ T$ is $\sigma\left(\mathscr{B}(S)^{*}\right.$, $\mathscr{B}(S)$ ) continuous on $\mathscr{B}(S)^{*}$. Now, we claim that (3) holds. For, if $\mu_{\alpha}$ and $\mu$ of $w^{*} M(\mathscr{B})$ are such that $\mu_{\alpha} \rightarrow \mu$ in $\tau_{w^{*}}$, then $\varphi_{\mu_{\alpha}} \rightarrow \varphi_{\mu}$ in $\sigma\left(\mathscr{B}(S)^{*}, \mathscr{B}(S)\right)$ (Proposition 2.6), and therefore

$$
\Phi\left(T_{\mu_{\alpha}} f\right)=\Phi \circ T\left(\varphi_{\mu_{\alpha}}\right) \longrightarrow \Phi \circ T\left(\varphi_{\mu}\right)=\Phi\left(T_{\mu} f\right)
$$

Since $\Phi$ is arbitrary in $\iota(\mathscr{B})^{*}$, we have $T_{\mu_{\alpha}} f \rightarrow T_{\mu} f$ in $\sigma\left(\iota(\mathscr{B}), \iota(\mathscr{B})^{*}\right)$. Thus (3) holds.

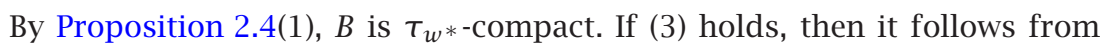
(3.7) that $R_{S} f$ is relatively weakly compact in $\iota(\mathscr{B})$. Thus (1) holds. So, (1) and (3) are equivalent.

Similarly, we show that (2) and (4) are equivalent. Next, we show that (1) and (3) imply (5).

Let $\mu, v \in w^{*} M(\mathscr{B})$ and $\varphi_{v}, \varphi_{\mu} \in M(\mathscr{B}(S))$ be as in (2.2). Since $f$ is in $\mathscr{W} \mathscr{A}(S, \mathscr{X}), f(\cdot)\left(x^{*}\right)$ is in $\mathscr{W} \mathscr{A} \mathscr{P}(S)$ for all $x^{*} \in \mathscr{X}^{*}$. By Proposition 3.1(5),

$$
\varphi_{v}\left\{T_{\varphi_{\mu}}\left[f(\cdot)\left(x^{*}\right)\right]\right\}=\varphi_{\mu}\left\{U_{\varphi_{v}}\left[f(\cdot)\left(x^{*}\right)\right]\right\}
$$


It follows from (2.2) and (3.10) that

$$
\begin{aligned}
{\left[\nu\left(T_{\mu} f\right)\right]\left(x^{*}\right) } & =\varphi_{\nu}\left\{T_{\varphi_{\mu}}\left[f(\cdot)\left(x^{*}\right)\right]\right\} \\
& =\varphi_{\mu}\left\{U_{\varphi_{v}}\left[f(\cdot)\left(x^{*}\right)\right]\right\} \\
& =\left[\mu\left(U_{v} f\right)\right]\left(x^{*}\right) .
\end{aligned}
$$

Since $x^{*} \in \mathscr{L}^{*}$ is arbitrary, we have

$$
v\left(T_{\mu} f\right)=\mu\left(U_{v} f\right) \quad\left(\mu, v \in w^{*} M(\mathscr{B})\right) .
$$

Thus (5) holds.

Similarly, we show that (2) and (4) imply (5).

For $x^{*} \in \mathscr{L}^{*}$ and $\mu \in w^{*} M(\iota(\mathscr{B}))$, define $\mu \circ x^{*}: \iota(\mathscr{B}) \rightarrow \mathbb{C}$ by

$$
\mu \circ x^{*}(h)=[\mu(h)]\left(x^{*}\right) \quad(h \in \iota(\mathscr{B})) .
$$

Then, $\mu \circ x^{*} \in \iota(\mathscr{B})^{*}$. Let $D=\left\{\mu \circ x^{*}: x^{*} \in \mathscr{X}^{*},\left\|x^{*}\right\|=1, \mu \in w^{*} M(\iota(\mathscr{B}))\right\}$. By the separation theorem, we show that $\overline{\mathrm{cCO}^{*}} w^{*} D=B_{\iota(\mathscr{B}) *}$, the unit ball of $\iota(\mathscr{B})^{*}$, where $w^{*}$ stands for $\sigma\left(\iota(\mathscr{B})^{*}, \iota(\mathscr{B})\right)$.

To show that (5) implies (4), we need to show that the mapping $\mu \rightarrow U_{\mu} f$ :

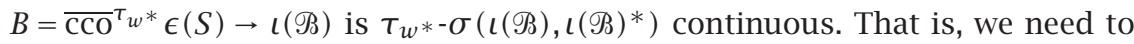
show that if $\mu_{\alpha} \subset B$ and $\mu \in B$ are such that $\mu_{\alpha} \rightarrow \mu$ in $\tau_{w^{*}}$, and if $F \in B_{\iota(\Re) * \text {, }}$ then

$$
F\left(U_{\mu_{\alpha}} f\right) \rightarrow F\left(U_{\mu} f\right)
$$

Note that $\overline{\operatorname{cco}}^{w^{*}} D$ is the unit ball of $\iota(\mathscr{B})^{*}$. For $v \circ x^{*} \in \operatorname{cco} D$, we have

$$
v \circ x^{*}\left[U_{\mu_{\alpha}} f\right] \longrightarrow v \circ x^{*}\left[U_{\mu} f\right]
$$

because it follows from (3.4) and (3.13) that

$$
\begin{aligned}
v \circ x^{*}\left[U_{\mu_{\alpha}} f\right] & =\left[v\left(U_{\mu_{\alpha}} f\right)\right]\left(x^{*}\right) \\
& =\left[\mu_{\alpha}\left(T_{v} f\right)\right]\left(x^{*}\right) \longrightarrow\left[\mu\left(T_{v} f\right)\right]\left(x^{*}\right) \\
& =\left[v\left(U_{\mu} f\right)\right]\left(x^{*}\right) \\
& =v \circ x^{*}\left(U_{\mu} f\right) .
\end{aligned}
$$

Let $A=\left\{U_{\mu} f: \mu \in B\right\}$. Then, $A$ is a bounded subset of $\iota(\mathscr{B})$. As in the discussion for Note (ii) and (iii) before Theorem 3 of [5], we regard $\operatorname{cco} D$ as a set of bounded function on $A$, that is, a subset of $\mathscr{B}(A)$. Then, the weak* closure 
$\overline{\mathrm{CCO}}^{w *} D$ in $\iota(\Re)^{*}$ is contained in the weak closure $\overline{\mathrm{CCO}}^{w} D$ in $\mathscr{B}(A)$. Since $\overline{\mathrm{CCO}}^{w} D$ is also norm closed in $\mathscr{B}(A)$, for $F \in \overline{\mathrm{CCO}}^{w *} D \subset \overline{\mathrm{CCO}}^{w} D$, we have a sequence of $\left\{v_{n} \circ x_{n}^{*}\right\}$ of $\operatorname{cco} D$ such that

$$
\left|F(h)-v_{n} \circ x_{n}^{*}(h)\right| \longrightarrow 0
$$

uniformly in $h \in A$. Since

$$
\begin{aligned}
\left|F\left(U_{\mu_{\alpha} f}\right)-F\left(U_{\mu} f\right)\right| \leq & \left|F\left(U_{\mu_{\alpha} f}\right)-v_{n} \circ x_{n}^{*}\left(U_{\mu_{\alpha}} f\right)\right| \\
& +\left|v_{n} \circ x_{n}^{*}\left(U_{\mu_{\alpha}} f\right)-v_{n} \circ x_{n}^{*}\left(U_{\mu} f\right)\right| \\
& +\left|v_{n} \circ x_{n}^{*}\left(U_{\mu} f\right)-F\left(U_{\mu} f\right)\right|,
\end{aligned}
$$

now (3.14) is a consequence of (3.15) and (3.17).

Similarly, we show that (5) implies (3). The proof is complete.

COROLlary 3.3. Let $S, \mathscr{X}$, and $\mathscr{A}$ be as in Theorem 3.2. Let $f \in \mathscr{A}$. Then, $T_{\mu} f \in \mathscr{A}$ for all $\mu \in M(\mathscr{A})$. Furthermore, if $f(S)$ is relatively weakly compact in $\mathscr{X}$, then $U_{\mu} f \in \mathscr{A}$ for all $\mu \in M(\mathscr{A})$.

Proof. As in the proof of (1) implying (3) of Theorem 3.2, we show the first statement. If $f(S)$ is relatively weakly compact in $\mathscr{X}$, then by the theorem, $L_{S} f$ is relatively weakly compact in $\mathscr{C}(S, \mathscr{C})$. Note that this time $f$ is in $\mathscr{A}$. We show the second statement as in the proof of (2) implying (4).

For every $x^{*} \in \mathscr{X}^{*},\left\|x^{*}\right\|=1$, and $s \in S$, define $x^{*} \circ s: \mathscr{C}(S, \mathscr{X}) \rightarrow \mathbb{C}$, by

$$
x^{*} \circ s(f)=x^{*}[f(s)] \quad(f \in \mathscr{C}(S, \mathscr{X})) .
$$

Then, $x^{*} \circ s \in \mathscr{C}(S, \mathscr{X})^{*}$, the dual space of $\mathscr{C}(S, \mathscr{X})$. Set $E=\left\{x^{*} \circ s: x^{*} \in\right.$ $\left.\mathscr{L}^{*},\left\|x^{*}\right\|=1, s \in S\right\}$. Let $B=\bar{E}^{w^{*}}$, where $w^{*}$ stands for the weak* topology $\sigma\left(\mathscr{C}(S, \mathscr{X})^{*}, \mathscr{C}(S, \mathscr{X})\right)$. Then, $B$ is weak* compact.

For every $f \in \mathscr{C}(S, \mathscr{X})$, define $\hat{f}: E \rightarrow \mathbb{C}$, by

$$
\hat{f}\left(x^{*} \circ s\right)=x^{*}[f(s)] \quad\left(x^{*} \circ s \in E\right) .
$$

We extend $\hat{f}$ from $E$ to $B$ continuously. So we have $\hat{f} \in \mathscr{C}(B)$.

Obviously, we have $\widehat{R_{t} f}$ for $t \in S$ and $R_{t} f$, that is, $\widehat{R_{t} f}\left(x^{*} \circ s\right)=x^{*}\left[R_{t} f(s)\right]$. The mapping $f \rightarrow \hat{f}: \mathscr{C}(S, \mathscr{X}) \rightarrow \mathscr{C}(B)$ is one to one and linear isometric. The space $\mathscr{C}(B)$ is a Banach space with norm topology. In the next theorem, we will also equip $\mathscr{C}(B)$ with the $P$-topology, the pointwise convergence topology. 
THEOREM 3.4. Let $S$ be a semitopological semigroup, and let $f \in \mathscr{C}(S, \mathscr{X})$ such that $f(S)$ is relatively weakly compact in $\mathscr{X}$. Then, the following statements are equivalent:

(1) $f \in \mathscr{W} \mathscr{A} \mathscr{P}(S, \mathscr{X})$;

(2) $L_{S} f$ is relatively weakly compact in $\mathscr{C}(S, \mathscr{X})$;

(3) $\widehat{R_{S} f}$ is relatively compact in $\mathscr{C}(B)$ in the $P$-topology;

(4) $\widehat{L_{S} f}$ is relatively compact in $\mathscr{C}(B)$ in the P-topology;

(5) $\lim _{m \rightarrow \infty} \lim _{n \rightarrow \infty} x_{m}^{*} f\left(s_{m} t_{n}\right)=\lim _{n \rightarrow \infty} \lim _{m \rightarrow \infty} x_{m}^{*} f\left(s_{m} t_{n}\right)$, whenever $\left\{x_{m}^{*}\right\} \subset \mathscr{X}^{*},\left\|x^{*}\right\|=1,\left\{t_{n}\right\},\left\{s_{m}\right\} \subset S$ and all the limits exist;

(6) $\lim _{m \rightarrow \infty} \lim _{n \rightarrow \infty} x_{m}^{*} f\left(t_{n} s_{m}\right)=\lim _{n \rightarrow \infty} \lim _{m \rightarrow \infty} x_{m}^{*} f\left(t_{n} s_{m}\right)$, whenever $\left\{x_{m}^{*}\right\} \subset \mathscr{X}^{*},\left\|x^{*}\right\|=1,\left\{t_{n}\right\},\left\{s_{m}\right\} \subset S$ and all the limits exist.

Proof. The equivalence of (1) and (2) comes from Theorem 3.2.

If (1) holds, then $R_{S} f$ is relatively weakly compact in $\mathscr{C}(S, \mathscr{X})$. Since the mapping $f \rightarrow \hat{f}$ is one to one and linear isometric, $\widehat{R_{S} f}$ is relatively weakly compact in $\mathscr{C}(B)$. So, $\widehat{R_{S} f}$ is relatively compact in $\mathscr{C}(B)$ in $P$-topology. Thus (3) holds.

Now, we show (3) implies (5). Let $\left\{x_{m}^{*}\right\} \subset \mathscr{X}^{*},\left\|x_{m}^{*}\right\|=1$, and $\left\{t_{n}\right\},\left\{s_{m}\right\} \subset S$ be sequences such that the iterated limits

$$
\lim _{m \rightarrow \infty} \lim _{n \rightarrow \infty} x_{m}^{*} f\left(s_{m} t_{n}\right), \quad \lim _{n \rightarrow \infty} \lim _{m \rightarrow \infty} x_{m}^{*} f\left(s_{m} t_{n}\right)
$$

exist. Thus, we have $\left\{\widehat{R_{t_{n}} f}\right\} \subset \mathscr{C}(B)$ and $\left\{x_{m}^{*} \circ s_{m}\right\} \subset E$. Let $\hat{g} \in \mathscr{C}(B)$ be $P$ topological cluster point of $\left\{\widehat{R_{t_{n}} f}\right\}$, and let $y \in B$ be cluster point of $\left\{x_{m}^{*} \circ s_{m}\right\}$. Then,

$$
\begin{aligned}
\lim _{m \rightarrow \infty} \lim _{n \rightarrow \infty} \widehat{R_{t_{n}} f}\left(x_{m}^{*} \circ s_{m}\right) & =\lim _{m \rightarrow \infty} \hat{g}\left(x_{m}^{*} \circ s_{m}\right) \\
& =\hat{g}(y)=\lim _{n \rightarrow \infty} \widehat{R_{t_{n}} f}(y) \\
& =\lim _{n \rightarrow \infty} \lim _{m \rightarrow \infty} \widehat{R_{t_{n}} f}\left(x_{m}^{*} \circ s_{m}\right) .
\end{aligned}
$$

Note that

$$
\begin{aligned}
& \lim _{m \rightarrow \infty} \lim _{n \rightarrow \infty} \widehat{R_{t_{n}} f}\left(x_{m}^{*} \circ s_{m}\right)=\lim _{m \rightarrow \infty} \lim _{n \rightarrow \infty} x_{m}^{*} f\left(s_{m} t_{n}\right), \\
& \lim _{n \rightarrow \infty} \lim _{m \rightarrow \infty} \widehat{R_{t_{n}} f}\left(x_{m}^{*} \circ s_{m}\right)=\lim _{n \rightarrow \infty} \lim _{m \rightarrow \infty} x_{m}^{*} f\left(s_{m} t_{n}\right) .
\end{aligned}
$$

Therefore, (5) holds.

That (5) implies (1) is a consequence of Grothendieck's double theorem [2, A.5]. Similarly, we show the equivalence among (2), (4), and (6). The proof is complete.

EXAMPLE 3.5. Let $S=\mathbb{N}=\{1,2, \ldots\}$, the semigroup of natural numbers. Let $l_{p}, 1<p<\infty$ be the usual sequence spaces with basis $\left\{e_{n}\right\}_{n=1}^{\infty}$, where $e_{n}=\left\{x_{k}\right\}_{k=1}^{\infty}$ with $x_{k}=1$ if $k=n$ and $x_{k}=0$ otherwise. Define $f: \mathbb{N} \rightarrow l_{p}$ by

$$
f(n)=e_{n} \quad(n \in \mathbb{N}) .
$$


Since $\mathbb{N}$ is abelian, $R_{S} f=L_{S} f$. For any subsequences $\left\{m_{i}\right\},\left\{n_{k}\right\}$ of $S$, and $\left\{\varphi_{i}\right\}$ of $l_{q}$ with $\|\varphi\| \nsucceq 1$, where $1 / p+1 / q=1$ and the norm being taken in the space $l_{q}$, we have that

$$
\begin{aligned}
& \lim _{i} \lim _{k} \varphi_{i}\left(f\left(m_{i}+n_{k}\right)\right)=\lim _{i} \lim _{k} \varphi_{i}\left(e_{m_{i}+n_{k}}\right)=\lim _{i} \lim _{k} \varphi_{i, m_{i}+n_{k}}=0, \\
& \lim _{k} \lim _{i} \varphi_{i}\left(f\left(m_{i}+n_{k}\right)\right)=\lim _{k} \lim _{i} \varphi_{i}\left(e_{m_{i}+n_{k}}\right)=\lim _{k} \lim _{i} \varphi_{i, m_{i}+n_{k}}=0,
\end{aligned}
$$

where $\varphi_{i, m_{i}+n_{k}}$ is the $\left(m_{i}+n_{k}\right)$ th component of $\varphi_{i}$. Therefore, by Theorem 3.4 we have $f \in \mathscr{W} \mathscr{A} \mathscr{P}\left(\mathbb{N}, l_{p}\right)$. However, $f(S)$ is not relatively norm compact but relatively weakly compact. Since $R_{S} f=L_{S} f$, the two orbits of $f$ are all relatively weakly compact. We note that $\mathbb{N}$ does not admit an identity.

REMARK 3.6. (a) The equivalence of (1) and (5) of Theorem 2.4 appeared in [5, Theorem 6]; though it was assumed that $S$ admits an identity, the proof of Theorem 6 did not use the identity. (b) In both Theorems 3.2 and 3.4, we assume that the range of $f(S)$ is relatively weakly compact to show that the relative weak compactness of $L_{S} f$ is equivalent to that of $R_{S} f$. We do not know if the condition of $f(S)$ is essential. We showed in [8, Corollary 8.4] that if $S$ admits an identity, then $f(S)$ is relatively weakly compact in $\mathscr{L}$ for all $f \in \mathscr{W} \mathscr{A} \mathscr{P}(S, \mathscr{X})$. Of course, if $\mathscr{X}$ is reflexive, then any bounded function has a relatively weakly compact range. (c) From the proof of Theorem 3.4, we see that to get the equivalence among (1), (3), and (5), it does not need the condition $f(S)$ being relatively weakly compact, neither does the equivalence among (2), (4), and (6).

ACKNOWLEDGMENTS. The research is supported in part by the National Science Foundations (NSF) of China and Harbin Institute of Technology (HIT).

\section{REFERENCES}

[1] B. Basit, Some problems concerning different types of vector valued almost periodic functions, Dissertationes Math. (Rozprawy Mat.) 338 (1995), 26.

[2] J. F. Berglund, H. D. Junghenn, and P. Milnes, Analysis on Semigroups. Function Spaces, Compactifications, Representations, Canadian Mathematical Society Series of Monographs and Advanced Texts, John Wiley \& Sons, New York, 1989.

[3] N. Dunford and J. T. Schwartz, Linear Operators. I. General Theory, Pure and Applied Mathematics, vol. 7, Interscience Publishers, New York, 1958.

[4] S. Goldberg and P. Irwin, Weakly almost periodic vector-valued functions, Dissertationes Math. (Rozprawy Mat.) 157 (1979), 46.

[5] P. Milnes, On vector-valued weakly almost periodic functions, J. London Math. Soc. (2) 22 (1980), no. 3, 467-472.

[6] W. M. Ruess and W. H. Summers, Ergodic theorems for semigroups of operators, Proc. Amer. Math. Soc. 114 (1992), no. 2, 423-432.

[7] C. Y. Zhang, Integration of vector-valued pseudo-almost periodic functions, Proc. Amer. Math. Soc. 121 (1994), no. 1, 167-174. 
[8]_ Vector-valued means and their applications in some vector-valued function spaces, Dissertationes Math. (Rozprawy Mat.) 334 (1994), 35.

[9] _ Vector-valued means and weakly almost periodic functions, Int. J. Math. Math. Sci. 17 (1994), no. 2, 227-237.

Chuanyi Zhang: Department of Mathematics, Harbin Institute of Technology, Harbin 150001, China

E-mail address: czhang@hope.hit . edu.cn 


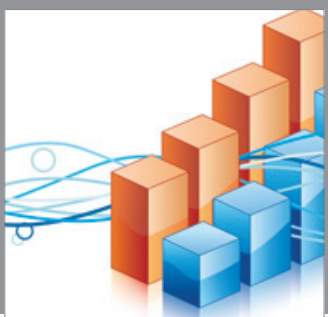

Advances in

Operations Research

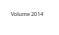

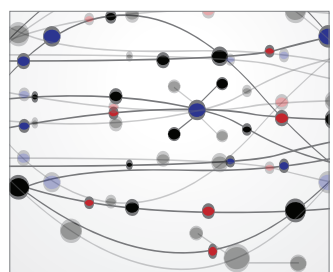

\section{The Scientific} World Journal
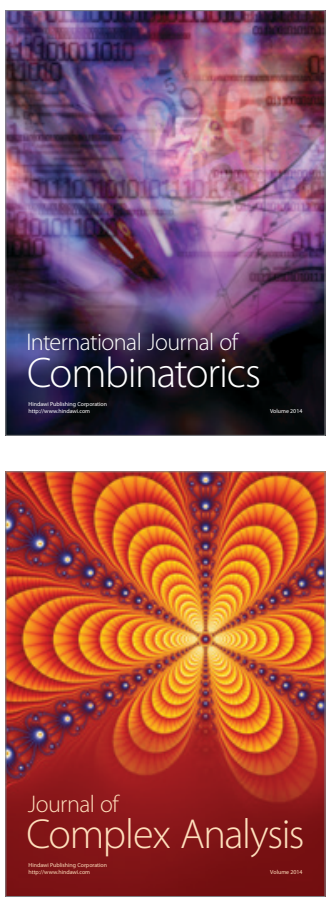

International Journal of

Mathematics and

Mathematical

Sciences
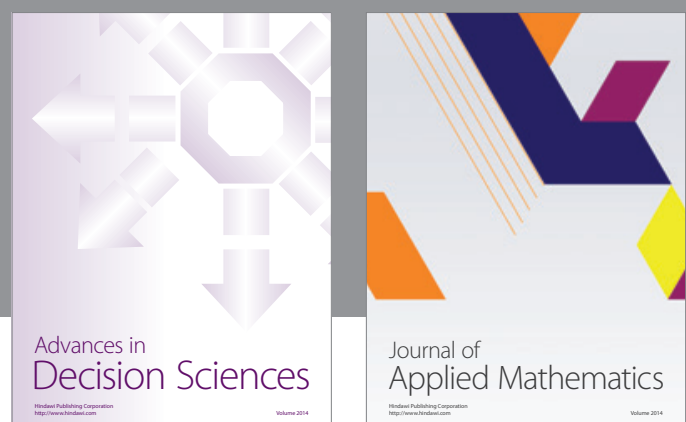

Journal of

Applied Mathematics
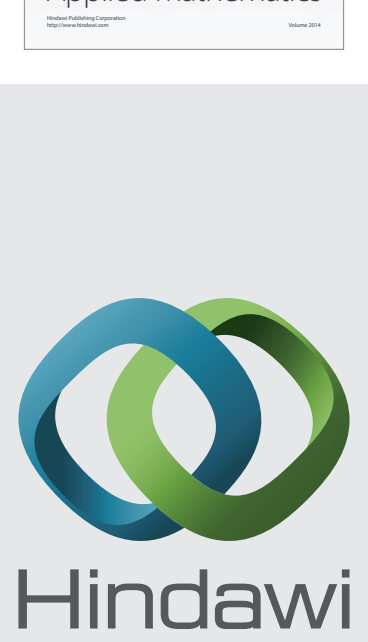

Submit your manuscripts at http://www.hindawi.com
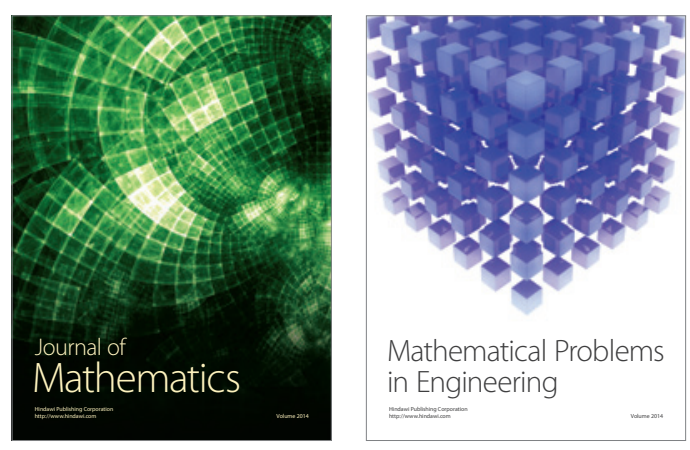

Mathematical Problems in Engineering
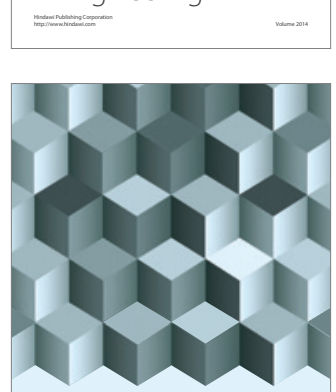

Journal of

Function Spaces
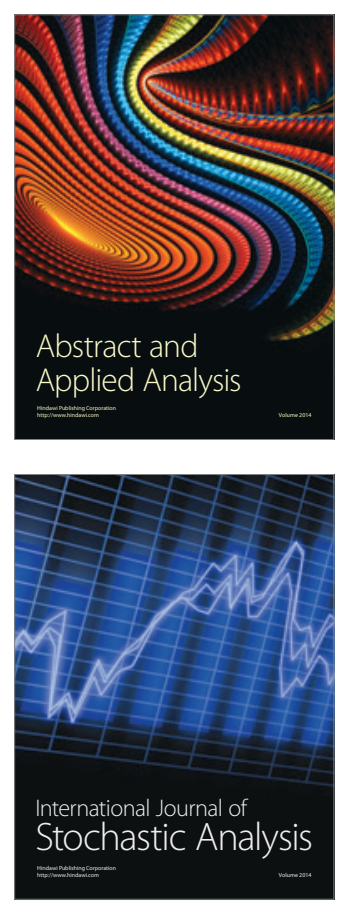

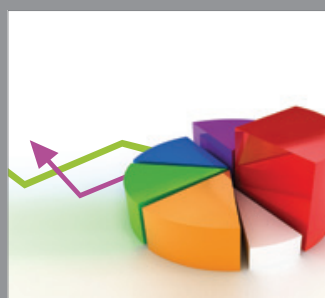

ournal of

Probability and Statistics

Promensencen
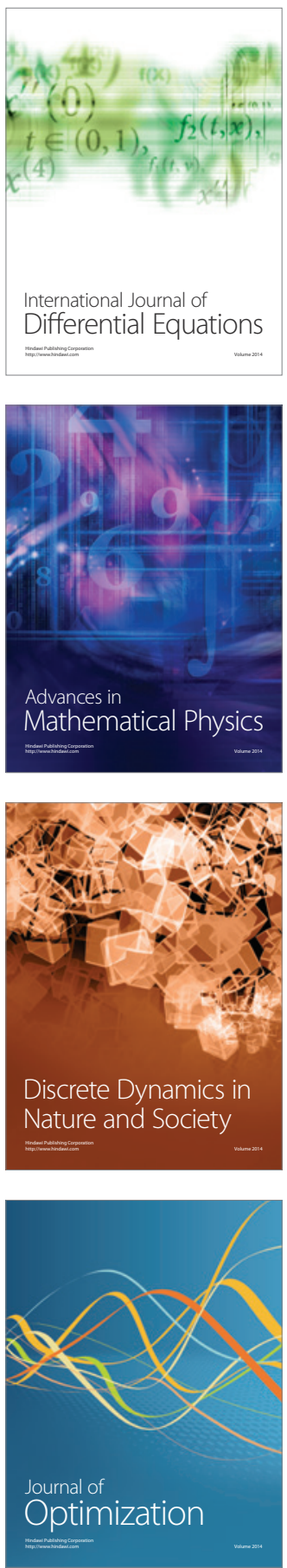